\title{
Visual Stabilization of Balance in Virtual Reality Using the HTC Vive
}

\author{
JONATHAN W. KELLY, BRENNA C. KLESEL, and LUCIA A. CHEREP, Iowa State University
}

\begin{abstract}
Vision in real environments stabilizes balance compared to an eyes-closed condition. For virtual reality to be safe and fully effective in applications such as physical rehabilitation, vision in virtual reality should stabilize balance as much as vision in the real world. Older virtual reality technology was previously found to stabilize balance but by less than half as much as real-world vision. Recent advancements in display technology might allow for vision in virtual reality to be as stabilizing as vision in the real world. This study evaluated whether viewing a virtual environment through the HTC Vive-a new consumer-grade head-mounted display-stabilizes balance, and whether visual stabilization is similar to that provided by real-world vision. Participants viewed the real laboratory or a virtual replica of the laboratory and attempted to maintain an unstable stance with eyes open or closed while standing at one of two viewing distances. Vision was significantly stabilizing in all conditions, but the virtual environment provided less visual stabilization than did the real environment. Regardless of the environment, near viewing led to greater visual stabilization than did far viewing. The smaller stabilizing influence of viewing a virtual compared to real environment might lead to greater risk of falls in virtual reality and smaller gains in physical rehabilitation using virtual reality.
\end{abstract}

Categories and Subject Descriptors: I.3.7 [Computer Graphics]: Three-Dimensional Graphics and Realism-Virtual reality; I.4.8 [Computer Applications]: Social and Behavioral Sciences-Psychology; H.5.1 [Information Systems]: Multimedia Information Systems-Artificial, Augmented, and Virtual realities; H.1.2 [Information Systems]: User Machine SystemsHuman Factors

General Terms: Virtual environments, Experimentation

Additional Key Words and Phrases: Balance, posture, stereoscopic displays, virtual environments

ACM Reference format:

Jonathan W. Kelly, Brenna C. Klesel, and Lucia A. Cherep. 2019. Visual Stabilization of Balance in Virtual Reality Using the HTC Vive. ACM Trans. Appl. Percept. 16, 2, Article 8 (March 2019), 11 pages.

https://doi.org/10.1145/3313902

\section{INTRODUCTION}

Virtual reality (VR) has proven to be a useful tool for applications including education, industry, psychological therapy, and physical rehabilitation. The popularity of VR has increased recently with the availability of lowcost head-mounted displays (HMD) marketed toward video game consumers. For VR to be a safe experience for users, and for it to be an effective tool for physical rehabilitation, it should ideally provide visual feedback about

This research was supported by a Seed Grant for Social Sciences from the Iowa State University College of Liberal Arts and Sciences. Authors' address: J. W. Kelly, B. C. Klesel, and L. A. Cherep, Department of Psychology, Iowa State University, W112 Lagomarcino Hall, 901 Stange Rd, Ames, IA, 50011-1041; emails: \{jonkelly, bklesel, lacherep\}@iastate.edu.

Permission to make digital or hard copies of all or part of this work for personal or classroom use is granted without fee provided that copies are not made or distributed for profit or commercial advantage and that copies bear this notice and the full citation on the first page. Copyrights for components of this work owned by others than ACM must be honored. Abstracting with credit is permitted. To copy otherwise, or republish, to post on servers or to redistribute to lists, requires prior specific permission and/or a fee. Request permissions from permissions@acm.org.

(c) 2019 Association for Computing Machinery.

1544-3558/2019/03-ART8 $\$ 15.00$

https://doi.org/10.1145/3313902 
self-motion that allows for stable balance. Visual information provided by the real environment reduces body sway, relative to an eyes-closed condition (Begbie 1967; Diener et al. 1984; Edwards 1946; Paulus et al. 1984; Witkin and Wapner 1950). Visual information provided by a virtual environment has a smaller stabilizing influence on balance compared to that provided by a real environment (Kelly et al. 2008). However, VR has advanced rapidly in recent years, and research showing that VR provides only modest stabilization of balance used VR systems that are now several generations old. Technological improvements in modern HMDs include lower latency, increased resolution, and increased field of view, all of which could lead to visual stabilization of balance on par with real world viewing. The current project was designed to evaluate whether a modern consumer-oriented VR system, the HTC Vive, can provide visual stabilization of balance comparable to real-world viewing.

In this study, participants attempted to stand on one foot while viewing the real laboratory environment or a virtual environment modeled after the laboratory. The one-foot stance was chosen because past work indicates that it leads to a detectable influence of vision on balance even when viewing a virtual environment (Kelly et al. 2008). Participants stood with eyes open and with eyes closed in order to quantify the stabilizing influence of vision on balance. Furthermore, distance to the nearest visible surface was manipulated (near vs. far) to determine whether the influence of viewing distance is similar in real and virtual environments.

No specific hypothesis was made regarding the comparison between real and virtual environments. It is unknown exactly why past work has shown a smaller influence of vision on balance when experienced through a virtual compared to real environment (Kelly et al. 2008), and therefore it is unknown whether modern systems have improved sufficiently to make visual information in a virtual environment as stabilizing as that from a real environment. Based on past work (Paulus et al. 1984; Dijkstra et al. 1992), it was expected that the near viewing distance would produce greater stabilization of balance than the far viewing distance, at least in the real environment.

To preview the results, viewing the virtual environment stabilized balance compared to eyes closed, but to a lesser extent than did viewing the real environment. The effect of viewing distance was similar in the real and virtual environments, with greater stability associated with the near viewing distance compared to the far viewing distance.

\section{METHOD}

\subsection{Participants}

Thirty-two undergraduate students (16 males, 16 females) at Iowa State University participated in exchange for course credit. Participants self-selected for the study and were advised when signing up for the study that they "must be comfortable standing in an unstable position (such as on one foot) for up to one minute at a time." The full study description advertised to participants is available on the Open Science Framework: https://osf.io/ 492wp. No measures of participant health or fitness were collected beyond the measures of balance described below. Average participant age was 19.78 years $(S D=1.93)$. All participants gave their informed consent prior to participation. The protocol was approved by the local institutional review board, and was therefore performed in accordance with the ethical standards specified by the 1964 Declaration of Helsinki.

\subsection{Stimuli}

Participants viewed the real surrounding lab and a virtual replica of the lab. The replica was geometrically equivalent to the real lab and included photographs of the real lab applied to the 3D scene. Participants stood facing a closed lab door, and a fixation point (a $3 \times 3 \mathrm{~cm}$ black square) was centered on the door at the participant's eye-height. Figure 1 shows the virtual scene.

When viewing the real environment, participants wore a plastic construction hat with a battery-powered LED light affixed to the top. The light consisted of a small battery box connected to the LED by a short flexible cord. When viewing the virtual environment, participants wore an HTC Vive HMD with the same battery-powered 


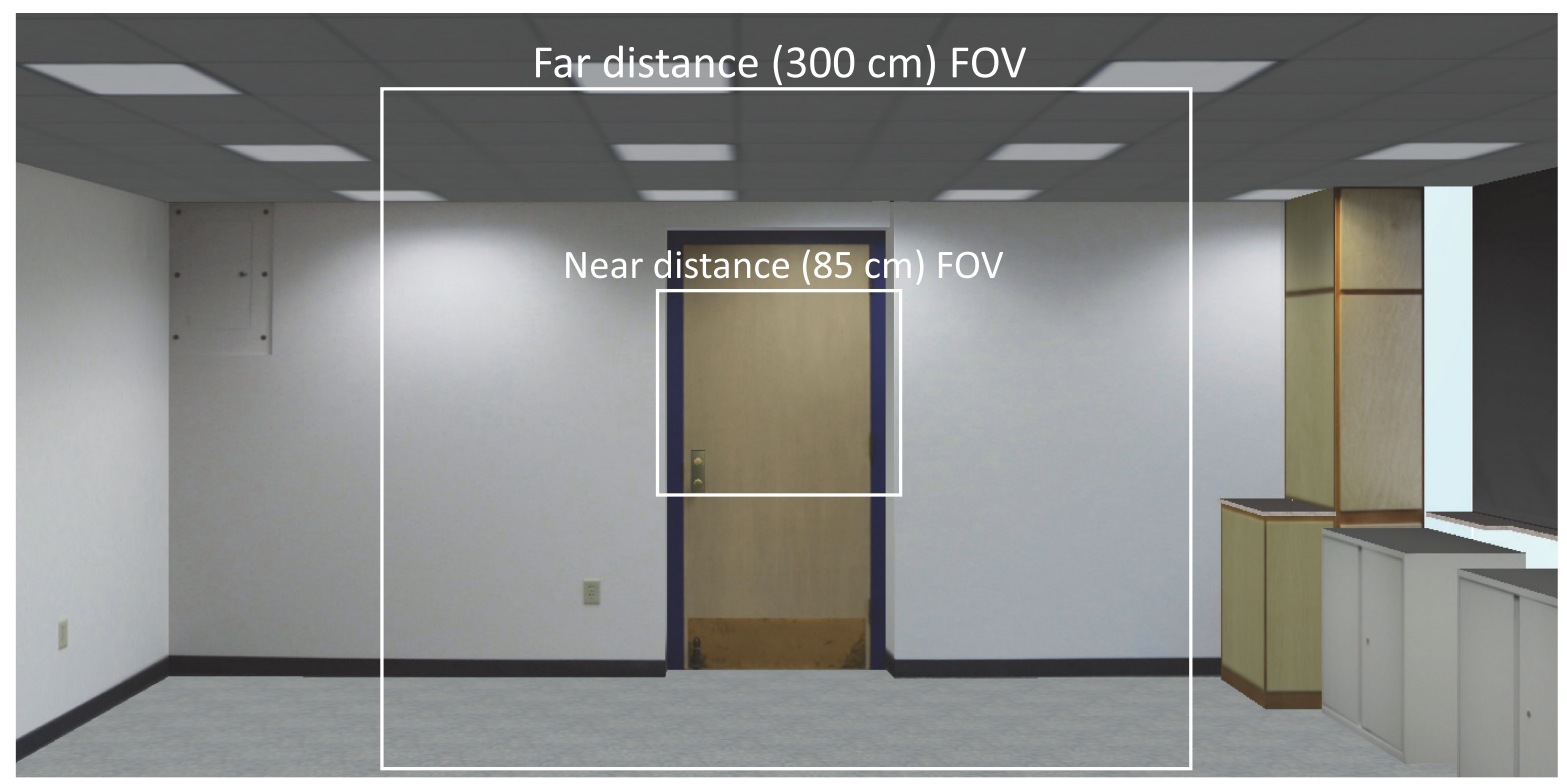

Fig. 1. Image of the virtual environment. White boxes indicate the approximate field of view boundaries when wearing the HTC Vive HMD at the two viewing distances.

LED affixed. The position of the light relative to the participant's head can impact measures of postural stability, and so care was taken to position the light at approximately the same location relative to the participant's head when wearing the HMD and the hat. During informal testing, the position of the light was measured when placed on each device and adjusted until the light position approximately matched across the two devices. Relative to the participant's body, the LED was approximately $15 \mathrm{~cm}$ above the participant's eyes.

The Vive HMD restricts the field of view to approximately $100^{\circ}$ horizontal by $110^{\circ}$ vertical. White boxes drawn in Figure 1 represent the approximate boundaries of the visible environment when wearing the Vive HMD while standing at the near and far viewing distances.

The virtual environment was displayed in stereo and graphics were generated on a Windows 10 computer with an Intel 6700K processor and Nvidia GeForce GTX 1070 graphics card. Head position and orientation was tracked using the Lighthouse tracking system bundled with the Vive HMD, and those data were used to update the graphics displayed in the HMD. Additionally, head position was tracked three-dimensionally with a fourcamera optical tracking system (PPTX; Worldviz). This tracking system measured the position of the batterypowered LED light worn on top of the participant's head, which was recorded at $90 \mathrm{~Hz}$ and served as the primary dependent measure.

\subsection{Design}

There were three independent variables, and all were manipulated within participants. First, the environment could be real or virtual. Second, the viewing distance (from the nearest wall) could be $85 \mathrm{~cm}$ (near distance) or $300 \mathrm{~cm}$ (far distance). Third, the participant's eyes could be open or closed. In this way, the study employed a $2 \times$ $2 \times 2$ design and included eight unique trials. Environment was blocked, such that participants completed all virtual trials in one block and all real trials in another block, and block order was counterbalanced. Within each environment block, viewing distance was also blocked and counterbalanced such that participants completed both near distance trials (eyes open and eyes closed) before moving to the far viewing distance, or vice versa. At 
each viewing distance, the order of eyes-open and eyes-closed trials was randomly determined. For each trial, the participant attempted to stand on one foot for 20 seconds. ${ }^{1}$

There are numerous ways to quantify stability and many of those measures are highly correlated. For example, Prieto et al. (1996) found root mean squared (RMS) distance, mean distance, range, and 95\% confidence ellipse area were highly correlated (Pearson's $r$ greater than 0.90). We follow the recommendation of Prieto et al. (1996) and analyze RMS distance as well as mean velocity, separately in the left-right and front-back body axes. RMS distance was calculated based on distance of the head from the mean head position on each trial, and is equivalent to standard deviation of the head position in this case. Velocity was derived from the head position data by dividing the distance of head displacement by the time elapsed between data points. The two measures could conceivably differ. For example, a person could remain within $1 \mathrm{~cm}$ of the start position but undergo rapid movement in order to maintain stability, leading to low RMS but high velocity. Alternatively, a person could slowly drift $5 \mathrm{~cm}$ to one side or the other, leading to high RMS but low velocity. For both measures, the statistical analyses focused on the eyes-open data, although eyes-closed data were also analyzed.

Comparison of eyes-open stability across real and virtual viewing may not tell the entire story. For example, if stability with eyes open is worse when viewing the virtual compared to real environment, this could be caused by a reduced stabilizing influence of vision in VR or by other factors that differ between conditions such as the HMD weight or the cable connecting the HMD to the computer, either of which could affect balance. Therefore, initial analyses focus on comparison across eyes-open conditions, but subsequent analyses use stability measures to compute Romberg quotients, calculated by dividing eyes closed by eyes open (Van-Parys and Njiokiktjien 1976) separately for each condition and each participant. A Romberg quotient greater than 1.0 indicates a stabilizing influence of vision.

\subsection{Procedure}

After completion of the informed consent process, the experimenter verbally provided an overview of the experimental procedures and the participant's task. The participant was instructed to balance on one foot for each 20 second trial. The participant was allowed to select either the left or right foot, with the condition that the same foot be used for all trials. The participant was instructed to remove shoes prior to the experiment, and to keep arms to the side during balance trials with explicit instruction to not cross arms or use the body for support (e.g., the participant was told not to rest one foot on top of the other). If necessary, the participant was allowed to touch the lifted foot to the ground briefly to regain balance, but the participant was told to restrict moving the standing leg except to avoid falling (doing so would inflate the standard deviation of head position on that trial). Trials on which the participant shifted the standing leg position were terminated and repeated until a successful trial was completed.

Prior to beginning the balance trials, the fixation point was adjusted to the participant's eye height. The participant was then guided to one of the two viewing distances, marked with tape to indicate placement of the participant's toes. Depending on condition, the participant donned either the hard hat or the HMD, and the LED light used for tracking head position was attached with Velcro. The participant was then instructed as to whether the upcoming trial required eyes open or closed. The experimenter then instructed the participant to stand on one foot and pressed a key to begin logging data for the trial. A bell indicated the end of the 20 -second trial, at which point the participant could stand naturally until the beginning of the next trial.

\section{RESULTS}

Figure 2 shows representative head position data from a single participant, separately for eyes-open and eyesclosed conditions as well as for the real and virtual environment.

\footnotetext{
${ }^{1}$ Recording duration varies in the literature, but 20 or 30 seconds is common. For example, 30 seconds is commonly used for side-by-side stance but 20 seconds for a more difficult tandem stance (Lihavainen et al. 2010). 


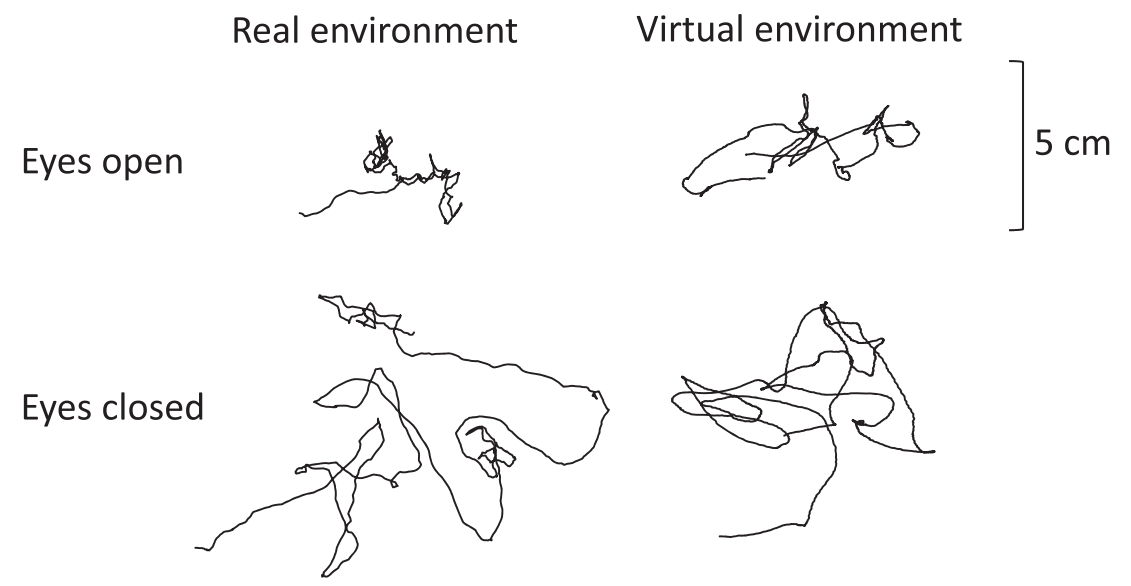

Fig. 2. Head position data from a representative participant in the near viewing distance condition. Romberg quotients based on the depicted head position data were 2.4 for the real environment and 1.7 for the virtual environment.

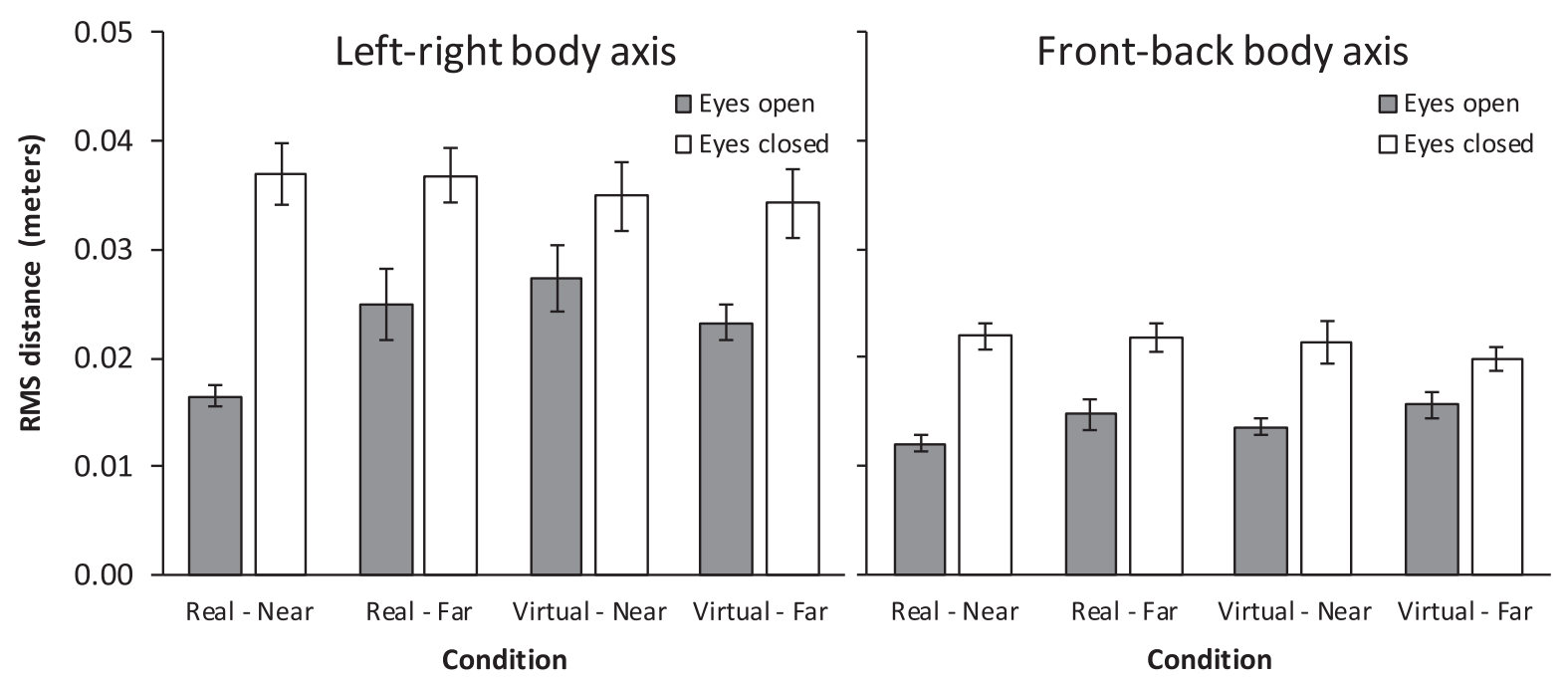

Fig. 3. Root mean squared (RMS) distance as a function of condition. Error bars represent \pm 1 standard error of the mean.

\subsection{RMS Distance}

Data were first analyzed by calculating RMS distance in the left-right and front-back body axes separately for each condition (Figure 3). RMS distance was analyzed in a repeated-measures Analysis of variance (ANOVA) with terms for environment (real or virtual) and viewing distance (near or far). Separate ANOVAs were conducted for the two body axes (left-right or front-back), and the focus was on eyes-open data. In the left-right body axis, the main effect of environment was statistically significant, $F(1,31)=4.263, p=.047, \eta_{p}^{2}=.121$, as was the interaction between environment and distance, $F(1,31)=6.226, p=.018, \eta_{p}^{2}=.167$. The main effect of distance was not significant. The interaction was due to higher RMS (i.e., lower stability) in the virtual compared to real environment, but only for the near viewing distance, $t(31)=3.256, p=.003$, and not for the far viewing distance, $t(31)=.482, p=.633$. The same analysis in the front-back body-axis revealed only a significant main effect of distance, $F(1,31)=7.556, p=.010, \eta_{p}^{2}=.196$, with higher RMS at the far compared to near viewing distance. 


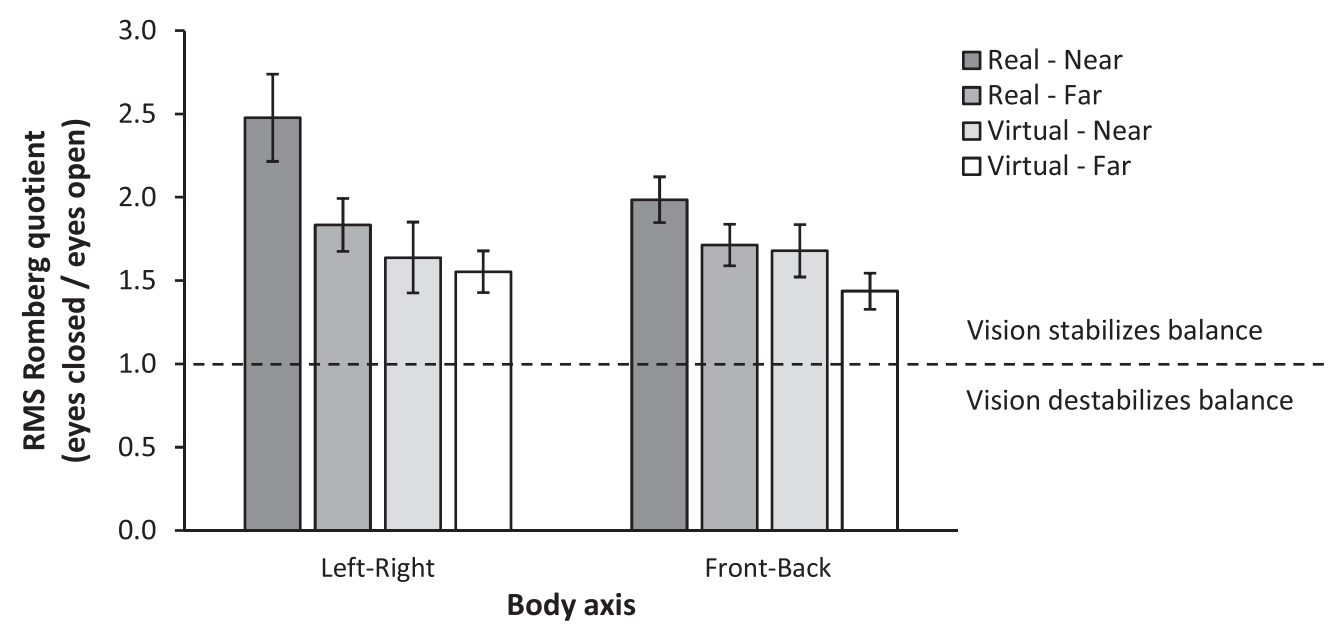

Fig. 4. Mean RMS-based Romberg quotients for each condition. Values greater than 1.0 indicate a stabilizing influence of vision on balance. Error bars represent \pm 1 standard error of the mean.

The eyes-closed data were also analyzed using repeated measures ANOVAs. The focus with eyes-closed data was whether physical characteristics of the HMD changed stability in the absence of vision. There were no significant main effects or interactions in either body axis, although there was a non-significant trend toward slightly lower RMS in the virtual compared to real conditions for both body axes.

All of the experimental conditions produced RMS-based Romberg quotients that were significantly greater than $1.0(p \leq .005)$, indicating a stabilizing influence of vision in all conditions. Romberg quotients (Figure 4) were analyzed in a repeated-measures ANOVA with terms for environment (real or virtual) and viewing distance (near or far). Separate ANOVAs were conducted for the two body axes (left-right or front-back).

For left-right body sway, the main effect of environment was statistically significant, $F(1,31)=27.476, p<$ $.001, \eta_{p}^{2}=.470$, with larger Romberg quotients in the real environment $(M=2.311, S E=0.128)$ compared to the virtual environment $(M=1.542, S E=0.091)$. The main effect of viewing distance was also significant, $F(1,31)=$ $6.072, p=.019, \eta_{p}^{2}=.164$, with larger Romberg quotients in the near viewing condition $(M=2.051, S E=0.104)$ compared to the far viewing condition $(M=1.802, S E=0.090)$. The interaction was not significant.

For front-back body sway, the main effect of environment was statistically significant, $F(1,31)=27.692, p<$ $.001, \eta_{p}^{2}=.472$, with larger Romberg quotients in the real environment $(M=1.920, S E=0.071)$ compared to the virtual environment $(M=1.399, S E=0.075)$. The main effect of viewing distance and the interaction were non-significant.

\subsection{Velocity}

Velocity was calculated separately for the left-right and front-back body axes and separately for each condition (Figure 5). Velocity was analyzed in a repeated-measures ANOVA with terms for environment (real or virtual) and viewing distance (near or far). Separate ANOVAs were conducted for the two body axes (left-right or front-back), and the focus was on eyes-open data. In the left-right body axis, the main effect of environment was statistically significant, $F(1,31)=9.912, p=.004, \eta_{p}^{2}=.242$, with greater velocity in the virtual $(M=.027, S E=.002)$ compared to real environment $(M=.021, S E=.002)$. The main effect of distance was also significant, $F(1,31)=7.278, p=$ $.011, \eta_{p}^{2}=.190$, with greater velocity at the far $(M=.027, S E=.002)$ compared to near viewing distance $(M=.022$, $S E=.001)$. The interaction was not significant. In the front-back body axis, the main effect of environment was significant, $F(1,31)=44.648, p<.001, \eta_{p}^{2}=.590$, with greater velocity in the virtual $(M=.023, S E=.001)$ compared 


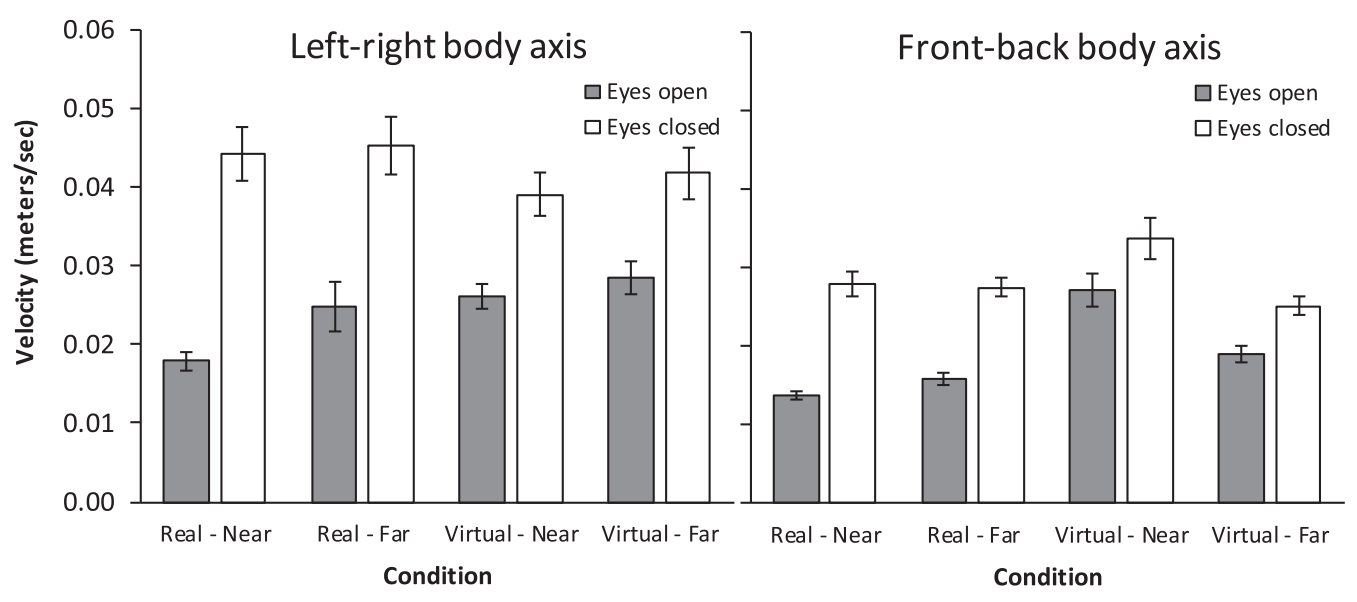

Fig. 5. Mean head velocity as a function of condition. Error bars represent \pm 1 standard error of the mean.

to real environment $(M=.015, S E=.001)$. The main effect of distance was also significant, $F(1,31)=9.762, p=$ $.004, \eta_{p}^{2}=.239$, with greater velocity at the near $(M=.020, S E=.001)$ compared to far viewing distance $(M=.017$, $S E=.001)$. The interaction between environment and distance was also significant, $F(1,31)=19.237, p<.001$, $\eta_{p}^{2}=.383$. The interaction was due to higher velocity for far compared to near viewing in the real environment, $t(31)=2.866, p=.007$, and lower velocity for far compared to near viewing in the virtual environment, $t(31)=$ $4.043, p<.007$.

The eyes-closed data were also analyzed using repeated measures ANOVAs. There were no significant main effects or interactions in the left-right body axis. In the front-back body axis, the main effect of distance was statistically significant, $F(1,31)=12.992, p=.001, \eta_{p}^{2}=.295$, as was the interaction between environment and distance, $F(1,31)=6.473, p=.016, \eta_{p}^{2}=.173$. The interaction was due to higher velocity at the near distance in the virtual environment compared to the other conditions.

All of the experimental conditions produced velocity-based Romberg quotients that were significantly greater than 1.0 ( $p \leq .002$ ), indicating a stabilizing influence of vision in all conditions. Romberg quotients (Figure 6) were analyzed in a repeated-measures ANOVA with terms for environment (real or virtual) and viewing distance (near or far). Separate ANOVAs were conducted for the two body axes (left-right or front-back).

For left-right body sway, the main effect of environment was statistically significant, $F(1,31)=9.198, p=.005$, $\eta_{p}^{2}=.229$, with larger Romberg quotients in the real environment $(M=2.155, S E=0.162)$ compared to the virtual environment $(M=1.596, S E=0.126)$. The main effect of viewing distance was also significant, $F(1,31)=5.037, p=$ $.032, \eta_{p}^{2}=.140$, with larger Romberg quotients in the near viewing condition $(M=2.058, S E=0.169)$ compared to the far viewing condition $(M=1.693, S E=0.099)$. The interaction was not significant.

For front-back body sway, the main effect of environment was statistically significant, $F(1,31)=4.320, p=$ $.046, \eta_{p}^{2}=.122$, with larger Romberg quotients in the real environment $(M=1.849, S E=0.095)$ compared to the virtual environment $(M=1.558, S E=0.110)$. The main effect of viewing distance was also significant, $F(1,31)=$ $4.554, p=.041, \eta_{p}^{2}=.128$, with larger Romberg quotients in the near viewing condition $(M=1.832, S E=0.109)$ compared to the far viewing condition $(M=1.575, S E=0.082)$. The interaction was not significant.

\section{DISCUSSION}

The analyses of eyes-open data based on RMS distance and velocity generally supported the conclusion that vision is more stabilizing when viewing a real compared to virtual environment. The RMS data indicated a real environment advantage in stability, but only for the near viewing distance and only in the left-right body axis. 


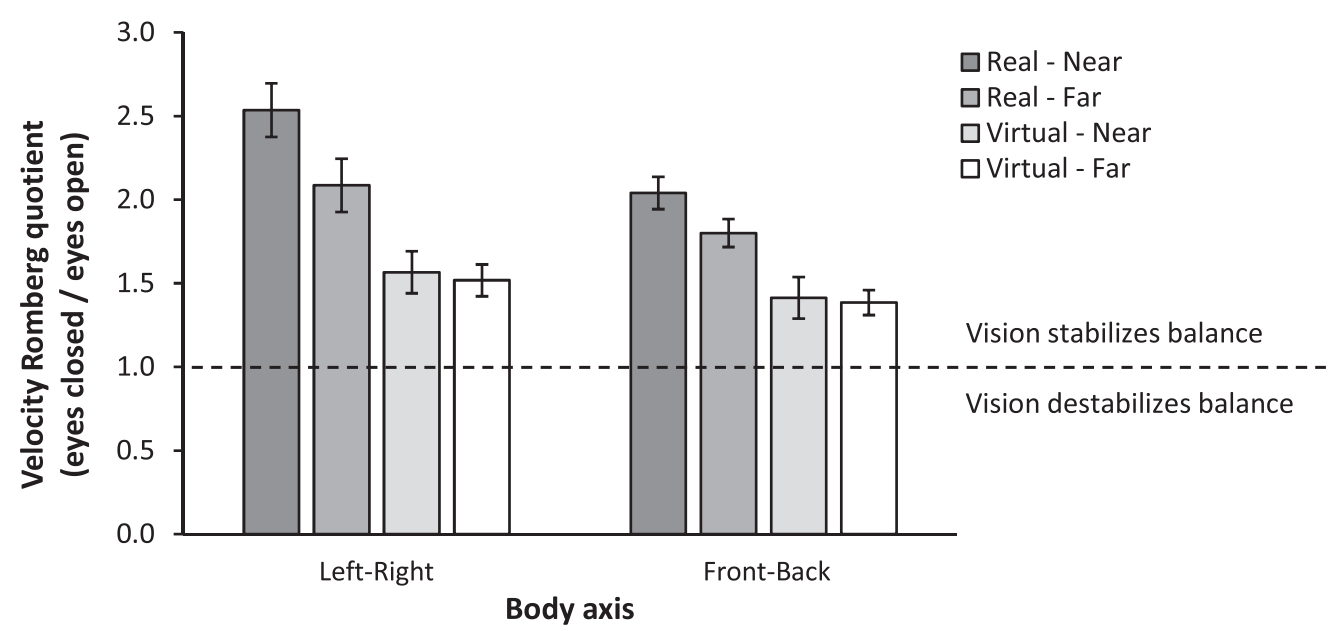

Fig. 6. Mean velocity-based Romberg quotients for each condition. Values greater than 1.0 indicate a stabilizing influence of vision on balance. Error bars represent \pm 1 standard error of the mean.

The velocity data indicated an overall real environment advantage regardless of viewing distance for both body axes.

The analyses of RMS-based and velocity-based Romberg quotients were generally consistent and supported three primary conclusions. First, vision stabilized balance in all conditions, including the VR condition using the HTC Vive. Second, viewing the virtual environment was less stabilizing than viewing the real environment. Third, vision was more stabilizing for the near compared to far viewing distance.

In general, the results based on RMS distance and velocity were consistent with those based on Romberg quotients computed from those same measures. Of course the main difference between those measures is that Romberg quotients are influenced by head movement in eyes-open as well as eyes-closed conditions. For example, although eyes-closed conditions did not significantly differ across the manipulated variables, there was a trend toward lower RMS in the virtual compared to real conditions (left-right body axis, real $M=.037, S E=0.002$, virtual $M=.035, S E=0.002$; front-back body axis, real $M=.022, S E=0.001$, virtual $M=.021, S E=0.001$ ). Such subtle differences in eyes-closed conditions only influence analysis of Romberg quotients. We view the conclusions based on RMS/velocity and Romberg quotients as complimentary. Whereas RMS and velocity indicate absolute postural stability, Romberg quotients indicate whether vision was stabilizing compared to no vision. Recent work on postural control (Lin et al. 2008) has emphasized dynamic patterns in body sway through detrended fluctuation analysis, and future work using such analyses might reveal further differences between real and virtual viewing.

The finding that viewing a virtual environment significantly stabilized balance compared to eyes closed (i.e., the Romberg data) replicates past work (Kelly et al. 2008). Other research in which the virtual environment was experimentally perturbed or manipulated also indicates an influence of vision on balance (Cunningham et al. 2006; Kelly et al. 2005; Stoffregen et al. 2004). It is unknown whether technological improvements associated with modern HMDs such as the HTC Vive have allowed for greater visual stabilization compared to older HMDs because none were tested in this study. Numerical comparisons with past work are not possible, since experimental differences such as the virtual environment (e.g., viewing distance) would confound such comparisons.

Although viewing the virtual environment did stabilize balance compared to eyes closed, it did so to a lesser extent than did viewing the real environment. This finding replicates past work (Kelly et al. 2008) and indicates that there are shortcomings to VR that have not been resolved in modern systems. It is unknown which 
technological limitations led to the smaller stabilizing influence of vision in VR, but likely candidates include reduced field of view relative to real-world viewing, as well as graphics latency and display quantization associated with VR.

The differences in balance when viewing real vs. virtual scenes may have implications for cybersickness or sickness induced by VR (Davis et al. 2014; Rebenitsch and Owen 2016). Cybersickness symptoms are commonly preceded by postural instability (Smart et al. 2002), and reduction of instability (e.g., by sitting instead of standing) can reduce cybersickness (Merhi et al. 2007). Therefore, it is possible that the increased body sway in virtual compared to real environments contributes to the experience of cybersickness, and reducing postural instability through technological improvements could be a direct path toward improving user experience in VR.

A recent meta-analysis evaluating the effectiveness of VR for physical rehabilitation separately considered studies that focused on improving gait, strength, motor control, and balance (Howard 2017). Compared to traditional rehabilitation programs, VR produced significantly greater improvement for gait and marginally greater improvement for strength and motor control, perhaps due to the increased enjoyment and motivation associated with using VR (Bryanton et al. 2006). In contrast, VR rehabilitation for balance was not significantly different from traditional rehabilitation. It is possible that the positive aspects of VR were offset by the relatively smaller stabilizing influence of viewing virtual compared to real environments.

These results may also have implications for other VR applications such as training. Examples of VR training programs include training of surgical skills (Piromchai et al. 2015) and machine assembly (Gavish et al. 2015). Disruption of the trainee's balance may lead to longer training times or failure to pass certain training milestones, especially those that require skilled motor coordination. Although post-training performance is often similar for VR and real-world training groups, VR training often takes longer (Carlson et al. 2015), which could be partially attributable to added task difficulty caused by postural instability.

Viewing distance affected visual control of balance in both real and virtual environments, such that close viewing led to greater visual stabilization compared to far viewing. This replicates past work (Paulus et al. 1984; Dijkstra et al. 1992) and indicates that manipulation of the visual scene can have similar effects in real and virtual environments.

Research on perception of distance indicates that perceived distance is foreshortened in virtual compared to real environments (see Renner et al. (2013) for a review), although this difference is narrowing with more modern displays (Buck et al. 2018; Creem-Regehr et al. 2015; Li et al. 2014; Kelly et al. 2017). Perception-action couplings have also been studied in the context of virtual environments (Kuhl et al. 2008; Mohler et al. 2007), but none to our knowledge have directly compared such couplings in matched real and virtual environments. To that end, the current project may be unique in highlighting deficiencies of perception-action coupling in virtual environments.

Additional research is needed to determine whether these results obtained with the HTC Vive will generalize to other popular displays that have been widely adopted. Relevant technical specifications differ dramatically across displays. For example, some stand-alone headsets such as the Samsung Gear VR and Oculus Go offer refresh rates of $60 \mathrm{~Hz}$, compared to $90 \mathrm{~Hz}$ found in the HTC Vive and Oculus Rift. Lower refresh rates could potentially lead to further destabilization of balance. Furthermore, most HMDs (including the HTC Vive) track position and orientation of the head, but some stand-alone headsets only track head orientation. The lack of graphical changes associated which changes in head position could cause additional destabilization of balance.

Lastly, the participants in this study were healthy and generally young adults. Therefore, more research is needed to determine whether these results generalize to other groups, such as older adults or skilled athletes.

In summary, viewing a virtual environment through the HTC Vive significantly stabilized balance but to a lesser extent than did viewing a real environment. The relatively smaller stabilizing influence of viewing a virtual compared to real environment might lead to greater risk of falls in VR and smaller gains in physical rehabilitation using VR. 


\section{OPEN PRACTICES}

All data have been made publicly available via the Open Science Framework and can be accessed at https://osf. io/492wp.

\section{ACKNOWLEDGMENTS}

We thank David Krummen, Lindsey Thompson, and Jonathan Anderson for assistance with data collection.

\section{REFERENCES}

G. H. Begbie. 1967. Some problems of postural sway. In Myotatic, Kinesthetic, and Vestibular Mechanisms, A. V. S. de Reuck and J. Knight (Eds.). Churchill, London, 80-92.

C. Bryanton, J. Bosse, M. Brien, J. Mclean, A. McCormick, and H. Sveistrup. 2006. Feasibility, motivation, and selective motor control: Virtual reality compared to conventional home exercise in children with cerebral palsy. Cyberpsychology and Behavior 9, 2 (2006), 123-128.

L. E. Buck, M. K. Young, and B. Bodenheimer. 2018. A comparison of distance estimation in HMD-based virtual environments with different HMD-based conditions. Transactions on Applied Perception 15, 3 (2018), 21:1-21:15.

P. Carlson, A. Peters, S. B. Gilbert, J. M. Vance, and A. Luse. 2015. Virtual training: Learning transfer of assembly tasks. IEEE Transactions on Visualization and Computer Graphics 21, 6 (2015), 770-782.

S. H. Creem-Regehr, J. K. Stefanucci, W. B. Thompson, N. Nash, and M. McCardell. 2015. Egocentric distance perception in the occulus rift (DK2). In Proceedings of the ACM Symposium on Applied Perception. ACM, New York City, NY, 47-50.

D. W. Cunningham, H. Nusseck, H. Teufel, C. Wallraven, and H. H. Blthoff. 2006. A psychophysical examination of swinging rooms, cylindrical virtual reality setups, and characteristic trajectories. In Proceedings of IEEE Virtual Reality. IEEE Computer Society, Washington D.C., 111118.

S. Davis, K. Nesbitt, and E. Nalivaiko. 2014. A systematic review of cybersickness. In Proceedings of the 2014 Conference on Interactive Entertainment. ACM, New York City, NY, 1-9.

H. C. Diener, J. Dichgans, M. Bacher, and B. Gompf. 1984. Quantification of postural sway in normals and patients with cerebellar diseases. Electroencephalography and Clinical Neurophysiology 57, 2 (1984), 134-142.

T. M. H. Dijkstra, C. C. A. Gielen, and B. J. M. Melis. 1992. Postural responses to stationary and moving scenes as a function of distance to the scene. Human Movement Science 11, 1-2 (1992), 195-203.

A. S. Edwards. 1946. Body sway and vision. Fournal of Experimental Psychology 36, 6 (1946), 526-535.

N. Gavish, T. Gutirrez, S. Webel, J. Rodrguez, M. Peveri, U. Bockholt, and F. Tecchia. 2015. Evaluating virtual reality and augmented reality training for industrial maintenance and assembly tasks. Interactive Learning Environments 23, 6 (2015), 778-798.

M. C. Howard. 2017. A meta-analysis and systematic literature review of virtual reality rehabilitation programs. Computers in Human Behavior 70 (2017), 317-327.

J. W. Kelly, B. B. Riecke, J. M. Loomis, and A. C. Beall. 2008. Visual control of posture in real and virtual environments. Perception and Psychophysics 70, 1 (2008), 158-165.

J. W. Kelly, L. A. Cherep, and Z. D. Siegel. 2017. Perceived space in the HTC vive. ACM Transactions on Applied Perception 15, 1 (2017), $2: 1-16$.

J. W. Kelly, J. M. Loomis, and A. C. Beall. 2005. The importance of perceived relative motion in the control of posture. Experimental Brain Research 161, 3 (2005), 285-292.

S. A. Kuhl, S. H. Creem-Regehr, and W. B. Thompson. 2008. Recalibration of rotational locomotion in immersive virtual environments. $A C M$ Transactions on Applied Perception 5, 3 (2008), 17:1-17:11.

B. Li, R. Zhang, and S. Kuhl. 2014. Minication affects action-based distance judgments in oculus rift HMDs. In Proceedings of the ACM Symposium on Applied Perception. ACM, New York City, NY, 91-94.

K. Lihavainen, S. Sipil, T. Rantanen, S. Sihvonen, R. Sulkava, and S. Hartikainen. 2010. Contribution of musculoskeletal pain to postural balance in community-dwelling people aged 75 years and older. The fournals of Gerontology: Series A 65A, 9 (2010), 990-996.

D. Lin, H. Seol, M. A. Nussbaum, and M. L. Madigan. 2008. Reliability of COP-based postural sway measures and age-related differences. Gait and Posture 28, 2 (2008), 337-342.

O. Merhi, E. Faugloire, M. Flanagan, and T. Stoffregen. 2007. Motion sickness, console video games, and head-mounted displays. Human Factors 45, 9 (2007), 920-935.

B. J. Mohler, W. B. Thompson, S. H. Creem-Regehr, P. Willemsen, H. L. Pick, and J. J. Rieser. 2007. Calibration of locomotion resulting from visual motion in a treadmill-based virtual environment. ACM Transactions on Applied Perception 4, 1 (2007), 4:1-4:15.

W. M. Paulus, A. Straube, and T. Brandt. 1984. Visual stabilization of posture: Physiological stimulus characteristics and clinical aspects. Brain 107, 4 (1984), 1143-1163.

P. Piromchai, A. Avery, M. Laopaiboon, G. Kennedy, and S. OLeary. 2015. Virtual reality training for improving the skills needed for performing surgery of the ear, nose or throat. Cochrane Database of Systematic Reviews (2015), 9.

ACM Transactions on Applied Perception, Vol. 16, No. 2, Article 8. Publication date: March 2019. 
T. E. Prieto, J. B. Myklebust, R. G. Hoffmann, E. G. Lovett, and B. M. Myklebust. 1996. Measures of postural steadiness: Differences between healthy young and elderly adults. IEEE Transactions on Biomedical Engineering 43, 9 (1996), 956-966.

L. Rebenitsch and C. Owen. 2016. Review on cybersickness in applications and visual displays. Virtual Reality 20, 2 (2016), $101-125$.

S. Renner, B. M. Velichkovsky, and R. Helmert. 2013. The perception of egocentric distances in virtual environments: A review. ACM Computer Surveys 46, 2 (2013), 23:1-40.

L. J. Smart, T. A. Stoffregen, and B. G. Bardy. 2002. Visually induced motion sickness predicted by postural instability. Hum. Factors 44, 3 (2002), 451-465.

T. A. Stoffregen, B. G. Bardy, O. Merhi, and O. Oullier. 2004. Postural responses to two technologies for generating optical flow. Presence: Teleoperators and Virtual Environments 13, 5 (2004), 601-615.

J. A. P. Van-Parys and C. H. J. Njiokiktjien. 1976. Romberg's sign expressed in a quotient. Agressologie 17 (1976), 95-100.

H. A. Witkin and S. Wapner. 1950. Visual factors in the maintenance of upright posture. American fournal of Psychology 63, 1 (1950), 31-50.

Received January 2018; revised January 2019; accepted February 2019 